\title{
Quantum critical dynamics of a qubit coupled to an isotropic Lipkin-Meshkov-Glick bath
}

\author{
H. T. Quan, ${ }^{1,2}$ Z. D. Wang, ${ }^{1}$ and C. P. Sun ${ }^{2}$ \\ ${ }^{1}$ Department of Physics and Center of Theoretical and Computational Physics, The University of Hong Kong, \\ Pokfulam Road, Hong Kong, China \\ ${ }^{2}$ Institute of Theoretical Physics, Chinese Academy of Sciences, Beijing, 100080, China
}

(Received 3 March 2007; published 9 July 2007)

\begin{abstract}
We explore a dynamic signature of quantum phase transition (QPT) in an isotropic Lipkin-Meshkov-Glick (LMG) model by studying the time evolution of a central qubit coupled to it. We evaluate exactly the timedependent purity, which can be used to measure quantum coherence, of the central qubit. It is found that distinctly different behaviors of the purity as a function of the parameter reveal clearly the QPT point in the system. It is also clarified that the present model is equivalent to an anti-Jaynes-Cummings model under certain conditions.
\end{abstract}

DOI: 10.1103/PhysRevA.76.012104

PACS number(s): 03.65.Yz, 05.70. $-\mathrm{a}, 05.90 .+\mathrm{m}$

\section{INTRODUCTION}

Quantum phase transitions (QPTs) [1] in spin systems, e.g., the $X Y$ model [2], the Lipkin-Meshkov-Glick (LMG) model [3], and the Dicke model [4], have aroused much interest in recent years. Most of these efforts have addressed possible connections of quantum entanglement measures, such as the concurrence, the entanglement entropy, and the negativity, with the QPTs in the systems. The scaling behavior $[2,5]$ of the entanglement demonstrate well the quantum criticality of these systems. On the other hand, being related to quantum measurement theory and quantum decoherence problems, theoretical studies of the bath influence on the dynamic property of a central system have also attracted much attention. Reference [6] claimed to find "an intrinsic limit to quantum coherence due to spontaneous symmetry breaking" in Lieb-Mattis model; the relationship between entanglement dynamics and paramagnet-ferromagnet phase transition was explored in Ref. [7]; it was shown in Ref. [8] that the Loschmidt echo decay enhanced at the critical point can be a signature of QPT in the transverse Ising model. Reference [9] shows that, in an exactly solvable model of decoherence, generic assumptions about the coupling strengths lead to a universal (Gaussian) suppression of coherence between pointer states. In fact, further deeper studies on the relevant issues of open quantum systems not only provides us a better understanding of the quantum-classical crossover, but also promises important potential applications in quantum-information processing [10].

In this paper, integrating coherently the above two interesting topics: Quantum phase transition and quantum open system [11], we elaborate how the QPT of the "bath" influences the dynamics of a central qubit coupled to it. It is shown that when the bath is in different phases, the purity of the central qubit exhibit distinctly different behaviors in two different phases. Moreover, it is also illustrated that under certain conditions our model is equivalent to an anti-JaynesCummings (anti-JC) model [12].

The paper is organized as follows. In Sec. II, we introduce the LMG model and summarize the main properties of this model. In Sec. III, we study the dynamic evolution of a central qubit coupled to a bath described by an isotropic LMG model, which is exactly solvable. In Sec. IV, we evaluate the purity of the central qubit in the symmetry broken phase and the symmetric phase, respectively. The QPT of the bath is well indicated by the behavior of the purity. In Sec. V, a connection between the current model and an anti-JC model is established. Section VI presents our summary and conclusion.

\section{LIPKIN-MESHKOV-GLICK (LMG) MODEL FOR QUANTUM PHASE TRANSITION}

We consider a central qubit (two-level system) that couples to a multispin bath, which is described by the LMG model [3]

$$
H_{B}=-\frac{\lambda}{N} \sum_{i<j}^{N}\left(\sigma_{i}^{x} \sigma_{j}^{x}+\gamma \sigma_{i}^{y} \sigma_{j}^{y}\right)-\sum_{i=1}^{N} \sigma_{i}^{z},
$$

where $\sigma_{i}^{\alpha}, \alpha=x, y, z(i=1,2, \ldots, N)$ are the Pauli matrices of the $i$ th spin, $\lambda / N$ denotes the coupling strength, which is inversely proportional to the spin number $N$. This Hamiltonian contains long-range interactions, i.e., every spin in the bath interacts with all the others. In the isotropic case, $\gamma=1$, the Hamiltonian is diagonal in the Dicke representation

$$
H_{B}=-\frac{2 \lambda}{N}\left[\mathbf{J}_{N}^{2}-\left(J_{N}^{z}\right)^{2}-\frac{N}{2}\right]-2 J_{N}^{z}
$$

and the ground state of $H_{B}$ lies in the subspace spanned by the Dicke states $\{|N / 2, M\rangle, M=-N / 2, \cdots N / 2\}$ [13]. Here, $\mathbf{s}$ $=\vec{\sigma} / 2, J_{N}^{\alpha}=1 / 2 \Sigma_{i=1}^{N} \sigma_{i}^{\alpha}$, and

$$
\begin{gathered}
\mathbf{J}_{N}^{2}\left|\frac{N}{2}, M\right\rangle=\frac{N}{2}\left(\frac{N}{2}+1\right)\left|\frac{N}{2}, M\right\rangle, \\
J_{N}^{z}\left|\frac{N}{2}, M\right\rangle=M\left|\frac{N}{2}, M\right\rangle .
\end{gathered}
$$

The eigenenergy corresponding to $|N / 2, M\rangle$ is $2 \lambda M^{2} / N$ $-2 M-\lambda N / 2$. Hence the ground state $|G\rangle$ is $\lambda$ dependent $[14]$, i.e., 


$$
|G\rangle=\left\{\begin{array}{lc}
\left|\frac{N}{2}, \frac{N}{2}\right\rangle \quad(0<\lambda<1), \\
\left|\frac{N}{2}, I(\lambda)\right\rangle \quad(\lambda>1),
\end{array}\right.
$$

where $I(\lambda)$ is the integer nearest to $N / 2 \lambda$. Equation (4) indicates that there is a level crossing at $\lambda=1$, which implies a QPT at $\lambda=1$ [1]. In the phases above and below $\lambda=1$ the properties of the ground state are significantly different, or the ground state of the system on two sides of $\lambda=1$ has different symmetries. Hence the point $\lambda=1$ is also a symmetry breaking point $[14,15]$ : When $0<\lambda<1$, the ground state of the bath is unique and fully polarized in the magnetic field direction, and thus the bath is in a symmetry broken phase; when $\lambda>1$, the ground state is infinitely degenerate and thus the bath is in a symmetric phase. We below elaborate how the dynamic evolution of the purity [16] (a measure of quantum coherence) depends on the coupling strength between the central qubit and the bath; in particular, we observe that the purity shows distinctly different behaviors in the two phases, which may be used to reveal the QPT point in the bath.

\section{DYNAMICS OF A CENTRAL QUBIT COUPLED TO AN ISOTROPIC LMG MODEL}

A qubit-bath model is described by the total Hamiltonian $H=H_{B}+H_{S}+H_{S B}[17,18]$, where $H_{S}=-2 s_{z}$ is the free Hamiltonian of the central qubit $S$. $H_{S B}$ denotes the coupling between $S$ and the bath $B$. Specifically, the total Hamiltonian can be written as

$H=-\frac{\lambda}{N} \sum_{i<j}^{N}\left(\sigma_{i}^{x} \sigma_{j}^{x}+\sigma_{i}^{y} \sigma_{j}^{y}\right)-\sum_{i=1}^{N} \sigma_{i}^{z}+\lambda^{\prime} \sum_{i}^{N}\left(\sigma_{i}^{x} \sigma^{x}+\sigma_{i}^{y} \sigma^{y}\right)-\sigma^{z}$,

where $\sigma^{\alpha}, \alpha=x, y, z$, are the Pauli operators of the central qubit; $\lambda^{\prime}$ is the coupling strength between the central qubit and the bath. In the Dicke representation, the above Hamiltonian can be rewritten as

$$
H=-\frac{\lambda}{N}\left[J_{N}^{+} J_{N}^{-}+J_{N}^{-} J_{N}^{+}-N\right]-2 J_{N}^{z}-2 \lambda^{\prime}\left(s_{+} J_{N}^{-}+s_{-} J_{N}^{+}\right)-\left(2 s_{z}\right),
$$

where $J_{N}^{ \pm}=J_{N}^{x} \pm i J_{N}^{y}$ and $s_{ \pm}=s^{x} \pm i s^{y}$ are the ladder operators of the $N$-spin bath and the central qubit, respectively. For simplicity, we denote the two eigenstate of the central qubit as $|\uparrow\rangle=|1 / 2,1 / 2\rangle$ and $|\downarrow\rangle=|1 / 2,-1 / 2\rangle$, and $s_{z}|\uparrow\rangle=|\uparrow\rangle / 2$, $s_{z}|\downarrow\rangle=-|\downarrow\rangle / 2$. In an invariant subspace $\mathcal{H}_{\mathcal{M}}$ of $H$ spanned by the ordered basis vector $\{|N / 2, M\rangle \otimes|\uparrow\rangle,|N / 2, M+1\rangle$ $\otimes|\downarrow\rangle\}$, the total Hamiltonian can be expressed as a quasidiagonal matrix with the diagonal blocks

$$
H_{M}=\left[\begin{array}{cc}
\alpha, & \zeta \\
\zeta, & \beta
\end{array}\right]
$$

where

$$
\begin{gathered}
\alpha=-\frac{\lambda}{2 N}\left[N^{2}-4 M^{2}\right]-2 M-1, \\
\beta=-\frac{\lambda}{2 N}\left[N^{2}-4(M+1)^{2}\right]-2(M+1)+1, \\
\zeta=-\lambda^{\prime} \sqrt{N(N+2)-4 M(M+1)} .
\end{gathered}
$$

A straightforward calculation determines the two eigenvalues $x_{1}$ and $x_{2}$ of $H_{M}$ as

$$
\begin{aligned}
& x_{1}=\frac{1}{2}\left[(\alpha+\beta)+\sqrt{(\alpha-\beta)^{2}+4 \zeta^{2}}\right], \\
& x_{2}=\frac{1}{2}\left[(\alpha+\beta)-\sqrt{(\alpha-\beta)^{2}+4 \zeta^{2}}\right],
\end{aligned}
$$

and the eigenstate $\left|\Psi_{1}\right\rangle$ corresponding to $x_{1}$ is

$$
\left|\Psi_{1}\right\rangle=a\left|\frac{N}{2}, M\right\rangle \otimes|\uparrow\rangle+b\left|\frac{N}{2}, M+1\right\rangle \otimes|\downarrow\rangle,
$$

where

$$
\begin{aligned}
& a=\frac{\zeta}{\sqrt{\left(\alpha-x_{1}\right)^{2}+\zeta^{2}}}, \\
& b=\frac{x_{1}-\alpha}{\sqrt{\left(\alpha-x_{1}\right)^{2}+\zeta^{2}}} .
\end{aligned}
$$

We would like to point out that, in the symmetric phase, all $x_{1}, x_{2}, a$, and $b$ are functions of $I(\lambda)$, i.e., $F=F[I(\lambda)]$ with $F=x_{1}, x_{2}, a$, and $b$. The dynamic evolution operator $U(t)$ $=\exp [-i H t]$ in the subspace $\mathcal{H}_{\mathcal{M}}$ can be expressed in terms of $x_{1}, x_{2}, a$, and $b$

$$
U_{M}(t)=\left[\begin{array}{ll}
a^{2} e^{-i x_{1} t}+b^{2} e^{-i x_{2} t}, & a b\left(e^{-i x_{1} t}-e^{-i x_{2} t}\right) \\
a b\left(e^{-i x_{1} t}-e^{-i x_{2} t}\right), & b^{2} e^{-i x_{1} t}+a^{2} e^{-i x_{2} t}
\end{array}\right],
$$

We wish to mention that the above dynamic evolution (11) is valid only for the cases when $-N / 2 \leq M<N / 2$, because $\quad\{|N / 2, M\rangle \otimes|\uparrow\rangle,|N / 2, M+1\rangle \otimes|\downarrow\rangle\} \quad$ is a twodimensional invariant subspace for these cases. But for the case $M=N / 2,\{|N / 2, M\rangle \otimes|\uparrow\rangle\}$ is a one-dimensional invariant subspace, i.e., $|N / 2, N / 2\rangle \otimes|\uparrow\rangle$ is an eigenstate of the total Hamiltonian (6), and its corresponding eigenenergy is $-(N+1)$. Thus the dynamic evolution of this state $|N / 2, N / 2\rangle \otimes|\uparrow\rangle$ is different from Eq. (11). We will discuss this point in the next section.

\section{PURITY OF THE CENTRAL QUBIT AS A WITNESS OF QUANTUM PHASE TRANSITION}

Based on the above results, we now solve the Schrödinger equation that describes the dynamics of the purity of the central qubit. To highlight the influence of the QPT of the bath on the coupled central qubit, it is assumed that the bath and the central qubit are initially in the ground state $|G\rangle$ (4) and a pure superposition state $c_{\uparrow}|\uparrow\rangle+c_{\downarrow}|\downarrow\rangle$, respectively. 
The evolution of the total system (the bath plus the central qubit) is

$$
\left.\left|\Psi_{N+1}(t)\right\rangle=e^{-i H t}|G\rangle \otimes\left(c_{\uparrow} \uparrow \uparrow\right\rangle+c_{\downarrow}|\downarrow\rangle\right),
$$

and the reduced density matrix of the central qubit is

$$
\rho^{S}(t)=\operatorname{Tr}_{\mathrm{B}}\left|\Psi_{N+1}(t)\right\rangle\left\langle\Psi_{N+1}(t)\right|,
$$

where $\operatorname{Tr}_{\mathrm{B}}$ means tracing out the degree of freedom of the bath.

The purity $P$ of the central qubit is defined as

$$
P=\operatorname{Tr}_{S}\left\{\left[\rho^{S}(t)\right]^{2}\right\}=\left[\rho_{\uparrow \uparrow}^{S}(t)\right]^{2}+\left[\rho_{\downarrow \downarrow}^{S}(t)\right]^{2}+2\left|\rho_{\uparrow \downarrow}^{S}(t)\right|^{2},
$$

which can be used to measure the quantum coherence. For a pure state, the purity equals to unity, while for a mixed state the purity is less than unity. The decay of purity indicates the loss of quantum coherence [16].

\section{A. Purity of the central qubit in two phases}

\section{Symmetric phase}

When $\lambda>1$, the bath is in the symmetric phase and $I(\lambda)$ $<N / 2$. As mentioned above, we can apply the evolution matrix (11) to obtain the reduced density matrix $\rho^{S}(t)$ of the central qubit with the matrix elements defined by

$$
\begin{gathered}
\rho_{\uparrow \uparrow}^{S}(t)=\sqrt{\left[\left|c_{\uparrow}\right|^{2} f(\lambda, t)+\left|c_{\downarrow}\right|^{2} h(\lambda, t)\right]^{2}}, \\
\rho_{\downarrow \downarrow}^{S}(t)=\sqrt{\left[\left|c_{\downarrow}\right|^{2} g(\lambda, t)+\left|c_{\uparrow}\right|^{2} i(\lambda, t)\right]^{2}}, \\
\left|\rho_{\uparrow \downarrow}^{S}(t)\right|=\sqrt{\left|c_{\uparrow} c_{\downarrow}^{*}\right|^{2} g(\lambda, t) \times f(\lambda, t)}=\left|\rho_{\downarrow \uparrow}^{S}(t)\right|,
\end{gathered}
$$

where

$$
\begin{gathered}
f(\lambda, t)=a^{4}+b^{4}+2 a^{2} b^{2} \cos \left[\left(x_{1}-x_{2}\right) t\right], \\
g(\lambda, t)=\left(a^{\prime}\right)^{4}+\left(b^{\prime}\right)^{4}+2\left(a^{\prime}\right)^{2}\left(b^{\prime}\right)^{2} \cos \left[\left(x_{1}^{\prime}-x_{2}^{\prime}\right) t\right], \\
h(\lambda, t)=2\left(a^{\prime}\right)^{2}\left(b^{\prime}\right)^{2}\left\{1-\cos \left[\left(x_{1}^{\prime}-x_{2}^{\prime}\right) t\right]\right\}, \\
i(\lambda, t)=2 a^{2} b^{2}\left\{1-\cos \left[\left(x_{1}-x_{2}\right) t\right]\right\},
\end{gathered}
$$

and the parameters $x_{1}^{\prime}, x_{2}^{\prime}, a^{\prime}$, and $b^{\prime}$ are defined by $F^{\prime}$ $=F[I(\lambda)-1]$ with $F^{\prime}=x_{1}^{\prime}, x_{2}^{\prime}, a^{\prime}$, and $b^{\prime}$. This subtle change from $\left(x_{1}, x_{2}, a, b\right)$ to $\left(x_{1}^{\prime}, x_{2}^{\prime}, a^{\prime}, b^{\prime}\right)$ is due to the fact that $|G\rangle \otimes|\uparrow\rangle$ and $|G\rangle \otimes|\downarrow\rangle$ belong to two different invariant subspace $\mathcal{H}_{\mathcal{M}}$ and $\mathcal{H}_{\mathcal{M}-1}$. If, for simplicity, we assume that the central qubit is initially in the superposition state $(|\uparrow\rangle$ $+|\downarrow\rangle) / \sqrt{2}$, we obtain from Eq. (14) the exact expression of the purity of the central qubit

$P=\frac{1}{4}[f(\lambda, t)+h(\lambda, t)]^{2}+\frac{1}{4}[i(\lambda, t)+g(\lambda, t)]^{2}+\frac{1}{2} g(\lambda, t) f(\lambda, t)$.

\section{Symmetry broken phase}

When $0<\lambda<1$, the bath is in the symmetry broken phase and the ground state is the fully polarized state $|G\rangle$
$=|N / 2, N / 2\rangle .|G\rangle \otimes|\uparrow\rangle$ is an eigenstate of the total Hamiltonian (5), and its corresponding eigenenergy is $-(N+1)$. Thus the dynamic evolution of this state is $\exp [i(N+1) t]$. While the dynamic evolution of the other state $|G\rangle \otimes|\downarrow\rangle$ can be obtained following the way mentioned above Eq. (11) with $M=N / 2-1$. After a similar procedure, if the central qubit is initially prepared in the superposition state $(|\uparrow\rangle$ $+|\downarrow\rangle) / \sqrt{2}$, the exact expression of the purity in the symmetry broken phase can be written as

$$
P=\frac{1}{4}[1+\widetilde{h}(\lambda, t)]^{2}+\frac{1}{4} \widetilde{g}^{2}(\lambda, t)+\frac{1}{2} \widetilde{g}(\lambda, t),
$$

where

$$
\begin{gathered}
\tilde{g}(\lambda, t)=\widetilde{a}^{4}+\widetilde{b}^{4}+2 \widetilde{a}^{2} \widetilde{b}^{2} \cos \left[\left(\widetilde{x}_{1}-\widetilde{x}_{2}\right) t\right], \\
\tilde{h}(\lambda, t)=2 \widetilde{a}^{2} \widetilde{b}^{2}\left\{1-\cos \left[\left(\widetilde{x}_{1}-\widetilde{x}_{2}\right) t\right]\right\} .
\end{gathered}
$$

The new parameters $\tilde{x}_{1}, \tilde{x}_{2}, \tilde{a}$, and $\tilde{b}$ are given by $\tilde{F}$ $=F[N / 2-1]$ with $\widetilde{F}=\widetilde{x}_{1}, \widetilde{x}_{2}, \widetilde{a}$, and $\widetilde{b}$.

To obtain the exact result of the purity of the central qubit, we need to know the coupling strength $\lambda^{\prime}$ [19] between the central qubit and the bath. In different references, this coupling strength is treated differently. For example, this coupling strength was assumed to be inversely proportional to the spin number of the bath in Ref. [6], while it was assumed to be inversely proportional to the square root of the spin number in some other Refs. [7,17]. We will consider both the two cases and we hereafter denote the two cases with the above two different coupling strengths as cases I and II, respectively. Generally speaking, when the central qubit is identical to the spins of the bath, the coupling strength between the central qubit and the bath should be equal to the coupling strength between the spins of the bath. This is case $\mathrm{I}$, and we will elaborate it in subsection B. Besides case I, case II will also be discussed in subsection C. The calculation for case II is the same as that for case I except that $\lambda^{\prime}$ in case I is changed to $\sqrt{N} \lambda^{\prime}$. The two different coupling strengths will lead to different behaviors of the purity.

\section{B. The coupling strength inversely proportional to the spin number of the bath (case I)}

First let us consider case I. Similar to the coupling mechanism in Ref. [6], we assume that the coupling strength $\lambda^{\prime}$ is just the coupling strength $\lambda / N$ between any two spins of the bath. Since the coupling strength $\lambda / N$ between spins in the LMG model is inversely proportional to the spin number $N$, the system is extensive.

Figure 1 clearly shows that, in the symmetry breaking phase, the purity of the central qubit remains as a constant unity, i.e., the qubit preserves its quantum coherence all the time. The decay of purity becomes vanishingly small in the thermodynamic limit. While in the symmetric phase, the purity varies periodically, as shown in Fig. 2.

The constant purity $P=1$ of the central qubit for the symmetry broken phase of the bath can also be verified through another approach. The ground state of the LMG model in the 

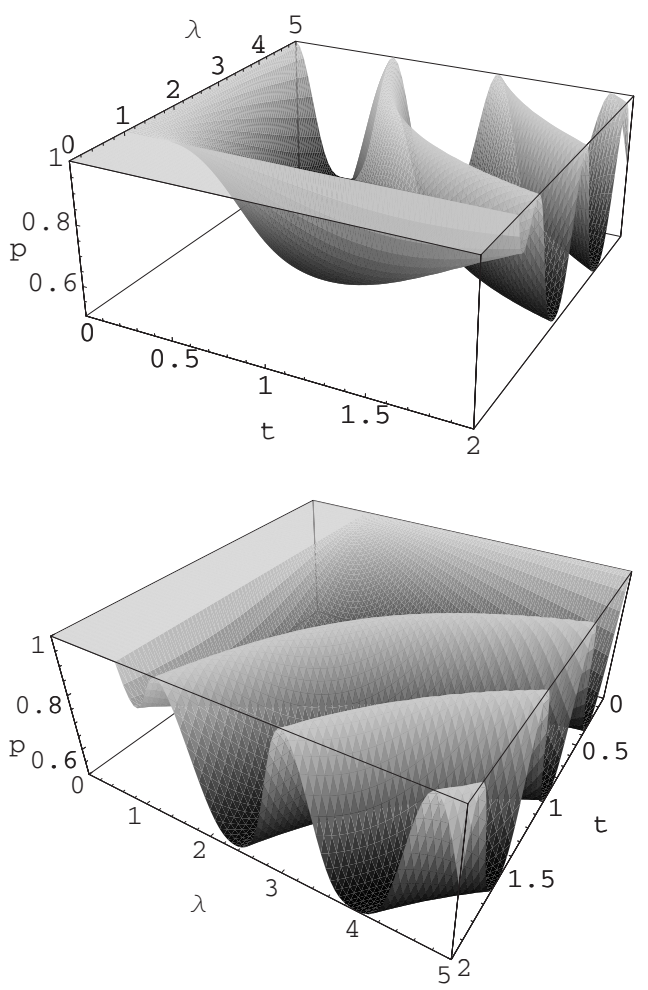

FIG. 1. Two different view angles on the dynamic evolution of the purity $P$ as functions of $\lambda$ and $t$ (case I). Here, $P$ and $\lambda$ are two dimensionless parameters, and $t \in[0,2 \mathrm{~s}]$. The gray level in this figure represents the value of the purity. The deeper in color, the smaller $P$. The QPT (symmetry breaking) at $\lambda=1$ is well signatured. The purity saturates when $N$ becomes large, i.e., there exist an asymptotic value of $P$ for every $\lambda$ in large $N$ limit. Here we have chosen the spin number of the environment $N=5000$.

symmetry broken phase $(0<\lambda<1)$ is $|G\rangle=|N / 2, N / 2\rangle$. The direct product of the bath $|G\rangle$ and the central qubit $c_{\uparrow}|\uparrow\rangle$ $+c_{\downarrow}|\downarrow\rangle$ can be expanded in the angular momentum coupling representation

$$
\begin{aligned}
|G\rangle \otimes\left(c_{\uparrow}|\uparrow\rangle+c_{2}|\downarrow\rangle\right) \approx & c_{\uparrow}\left|\frac{1}{2}(N+1), \frac{1}{2}(N+1)\right\rangle \\
& +c_{\downarrow}\left|\frac{1}{2}(N-1), \frac{1}{2}(N-1)\right\rangle,
\end{aligned}
$$

where we have used the Clebsch-Gordan (CG) coefficient

$$
\begin{aligned}
&\left|\frac{N}{2}, \frac{N}{2}\right\rangle \otimes|\uparrow\rangle=\left|\frac{1}{2}(N+1), \frac{1}{2}(N+1)\right\rangle, \\
&\left|\frac{N}{2}, \frac{N}{2}\right\rangle \otimes|\downarrow\rangle= \frac{\sqrt{N}}{\sqrt{N+1}}\left|\frac{1}{2}(N-1), \frac{1}{2}(N-1)\right\rangle \\
&+\frac{1}{\sqrt{N+1}}\left|\frac{1}{2}(N+1), \frac{1}{2}(N-1)\right\rangle .
\end{aligned}
$$

The total Hamiltonian (5) of the qubit and the bath can be rewritten as

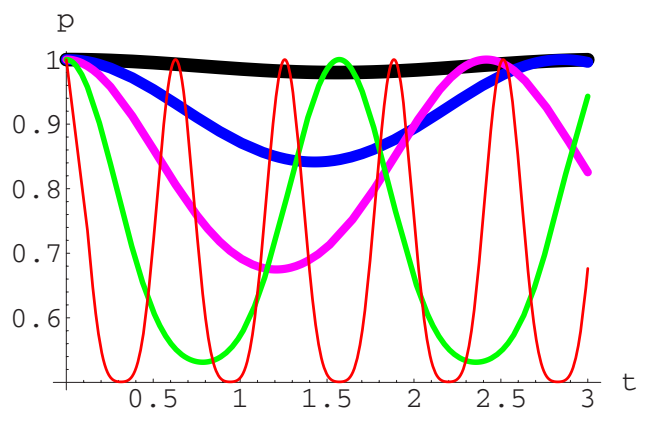

FIG. 2. (Color online) Dynamic evolution of the purity $P$ as a function of time $t(\in[0,3 \mathrm{~s}])$ for different $\lambda$. In the symmetry broken phase $(0<\lambda<1), P$ remains as a constant unity. In the symmetric phase $(\lambda>1), P$ ranges from 0.5 to 1 , and the period of the oscillation decreases as $\lambda$ increases. The curves with different thicknesses (from thick to thin) represent $\lambda=1.01, \lambda=1.1, \lambda=1.3, \lambda=2$, and $\lambda=5(N=5000)$.

$$
H=-\frac{\lambda}{N}\left[2 \mathbf{J}_{N+1}^{2}-2\left(J_{N+1}^{z}\right)^{2}-(N+1)\right]-2 J_{N+1}^{z} .
$$

Through the above approximation (19), both $|N / 2, N / 2\rangle$ $\otimes|\uparrow\rangle$ and $|N / 2, N / 2\rangle \otimes|\downarrow\rangle$ are the eigenstate of the total Hamiltonian with the eigenenergy $-(N+1)$ and $2 \lambda / N-(N$ $-1)$ respectively, and then the dynamic evolution of the two states are obvious. After a straightforward derivation, the reduced density matrix of the qubit is expressed as

$$
\rho^{S}(t)=\left|c_{\uparrow}\right|^{2}|\uparrow\rangle\left\langle\left.\uparrow|+| c_{\downarrow}\right|^{2} \mid \downarrow\right\rangle\left\langle\downarrow\left|+c_{\uparrow} c_{\downarrow}^{*} e^{i(2 \lambda / N+2) t}\right| \uparrow\right\rangle\langle\downarrow|+\text { H.c. }
$$

and the purity of $\rho^{S}(t)$ remains as unity by applying Eq. (14). It is thus proven that the qubit preserves its quantum coherence when the bath is in its symmetry broken phase $(0<\lambda$ $<1)$.

\section{The coupling strength inversely proportional to the square root of the spin number of the bath (case II)}

We now turn to case II, where the coupling strength between the central qubit and the bath is inversely proportional to the square root of the spin number of the bath $[7,17]$. Being different from case I, the central qubit does not preserve its coherence when the bath is in the symmetry broken phase (see Fig. 3), although the purity varies also periodically in the symmetric phase (Fig. 4). Nevertheless, both the range and the pattern of its time dependence in the two phases are different from those in case I. We also remark that the purity $P$ saturates in the symmetry broken phase when $N$ increases, just like that in the symmetric phase of case I; while the dynamic behavior of the purity in the symmetric phase depends on $N$ with the period being inversely proportional to $\sqrt{N}$ approximately, as analyzed later.

\section{EQUIVALENCE TO AN ANTI-JAYNES-CUMMINGS MODEL}

In this section we show the equivalence between the above model and an anti-JC model with an intensity- 

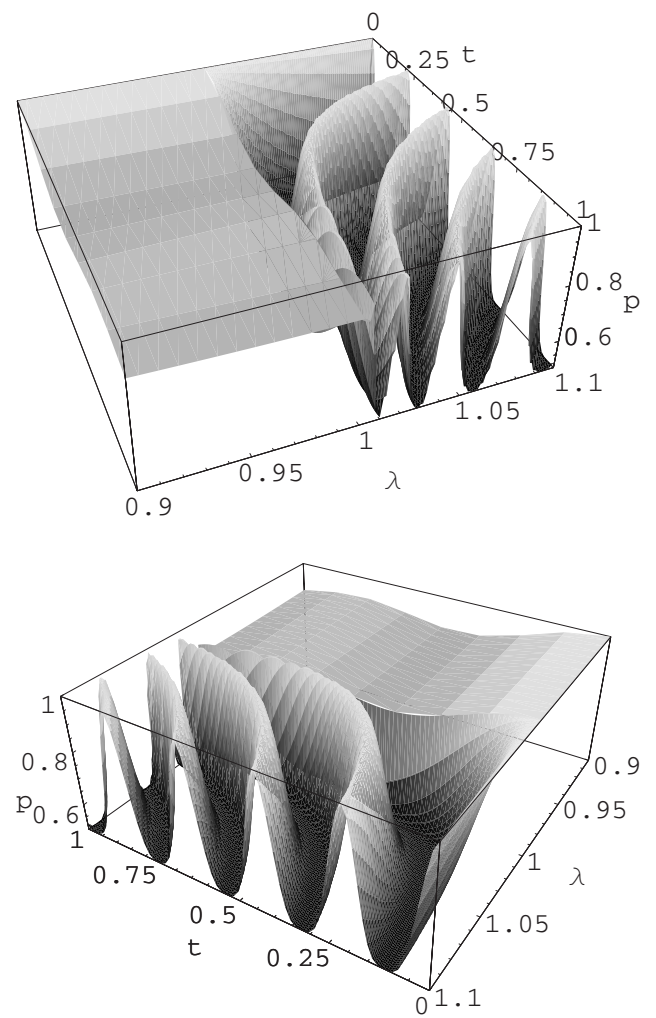

FIG. 3. Dynamic evolution of the purity $P$ as functions of $\lambda$ and $t$ (case II) in the symmetric phase and symmetry broken phase. The physical meanings of $P$ and $\lambda$ are the same as that in Fig. 1, and $t \in[0,1 \mathrm{~s}]$. Similar to Fig. 1 , the gray level in this figure also represents the value of the purity. Clearly the purity varies in distinctly different manners in the two phases, which may be considered as an indication of QPT at the critical point $\lambda=1$. The purity reaches a steady state in the symmetry broken phase, while the pattern of the purity will always change with $N$ in the symmetric phase. Here we choose $N=1000$.

dependent coupling strength [20]. With this observation, we can exactly solve the dynamical equation about time evolution.

\section{A. Symmetry broken phase}

When the bath is in the symmetry broken phase, our model may be recast into an anti-JC model. Actually the equivalence between the LMG model and Dike model was just studied recently [21].

In the symmetry broken phase $(0<\lambda<1)$, the ground state $|N / 2, N / 2\rangle$ of the bath corresponds to a low excitation Fock state $|0\rangle$ after the HP transformation. The mean photon number $n=\left\langle d^{\dagger} d\right\rangle=0$. Hence we can directly expand the Holstein-Primakoff (HP) transformation [22] to the firstorder [23]

$$
\begin{gathered}
J_{N}^{+}=\sqrt{N} d, \quad J_{N}^{-}=\left(J_{N}^{+}\right)^{\dagger} \\
J_{N}^{z}=N / 2-d^{\dagger} d,
\end{gathered}
$$

and the Hamiltonian (6) can be rewritten as

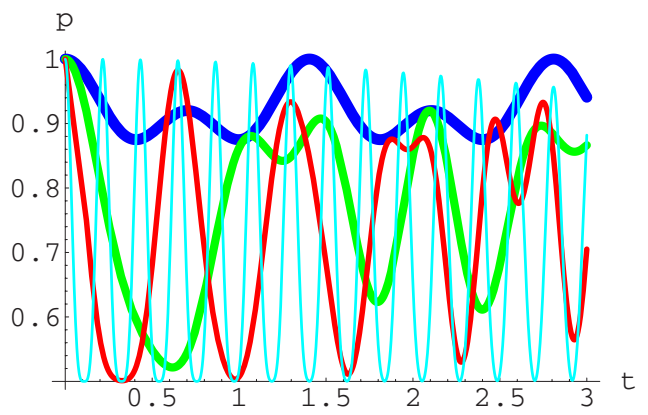

FIG. 4. (Color online) Dynamic evolution of the purity $P$ as a function of time $t(\in[0,3 \mathrm{~s}])$ for different $\lambda$. In the symmetry breaking phase $(0<\lambda<1)$, the dynamic behavior of $P$ saturates when $N$ increases, which ranges from about 0.88 to 1 with the period about 1.4. In the symmetric phase $(\lambda>1), P$ ranges from 0.5 to 1 , and the period of the oscillation decreases as $\lambda$ increases. The curves of different thicknesses (from thick to thin) represent $\lambda$ $=1.0005, \lambda=1.0015, \lambda=1.01$, and $\lambda=1.1(N=1000)$.

$$
H=2(1-\lambda) d^{\dagger} d-N-2 \lambda^{\prime} \sqrt{N}\left(s_{+} d^{\dagger}+s_{-} d\right)-2 s_{z} .
$$

Let us recall that the anti-JC Hamiltonian can be rewritten as

$$
H_{\mathrm{AJC}}=\nu d^{\dagger} d-k\left(\sigma_{+} d^{\dagger}+\sigma_{-} d\right)+\frac{1}{2} \omega \sigma_{z},
$$

where $d^{\dagger}$ and $d$ are the creation and annihilation operators of the single-mode quantized field with the frequency $\nu ;-k$ is the coupling strength between the field and the two-level system; $\omega$ is the level spacing between the two-level system; $\sigma_{+}=\left(\sigma_{x}+i \sigma_{y}\right) / 2$ and $\sigma_{-}=\left(\sigma_{x}-i \sigma_{y}\right) / 2$. Hence the model described by Eq. (23) is an anti-JC model (24) with $\nu=2(1$ $-\lambda), \omega=-2$, and $k=2 \lambda^{\prime} \sqrt{N}$. We now illustrate that the boson mode characterized by $d$ and $d^{\dagger}$ may be mapped from the collective spin $J$ in a low excitation limit. Note that the different mapping ways depend on the phases of the bath because the bosonization of collective spin is essentially a mean field approach based on choice of the order parameter.

The solution $|\Psi(t)\rangle$ of the Schrödinger equation $i \hbar \partial_{t}|\Psi(t)\rangle=H_{\mathrm{AJC}}|\Psi(t)\rangle$ can be expressed as

$$
|\Psi(t)\rangle=\sum_{n=0}\left[c_{\uparrow, n+1}(t)|\uparrow\rangle \otimes|n+1\rangle+c_{\downarrow, n}(t)|\downarrow\rangle \otimes|n\rangle\right],
$$

where $n=\left\langle d^{\dagger} d\right\rangle$ is the mean "photon" number. A straightforward calculation determines the probability amplitudes [12]

$$
\begin{aligned}
c_{\uparrow, n+1}(t)= & \left\{c_{\uparrow, n+1}(0)\left[\cos \left(\Omega_{n} t\right)-i \frac{\Delta}{\Omega_{n}} \sin \left(\Omega_{n} t\right)\right]\right. \\
& \left.+i \frac{k}{\Omega_{n}} \sqrt{n+1} c_{\downarrow, n}(0) \sin \left(\Omega_{n} t\right)\right\} \exp \left(i \frac{\Delta}{2} t\right),
\end{aligned}
$$




$$
\begin{aligned}
c_{\downarrow, n}(t)= & \left\{c_{\downarrow, n}(0)\left[\cos \left(\Omega_{n} t\right)+i \frac{\Delta}{\Omega_{n}} \sin \left(\Omega_{n} t\right)\right]\right. \\
& \left.+i \frac{k}{\Omega_{n}} \sqrt{n+1} c_{\uparrow, n+1}(0) \sin \left(\Omega_{n} t\right)\right\} \exp \left(-i \frac{\Delta}{2} t\right),
\end{aligned}
$$

where $\Delta=\nu+\omega$ and $\Omega_{n}=\sqrt{(\Delta / 2)^{2}+k^{2}(n+1)}$. After a routine calculation, we obtain the purity of the central qubit (with an initial state $(|\uparrow\rangle+|\downarrow\rangle) / \sqrt{2})$ in the symmetry broken phase $(0<\lambda<1)$ (see Appendix A)

$$
\begin{aligned}
P= & \frac{1}{4}\left[1+\cos ^{2}\left(\Omega_{0} t\right)+\left(\frac{\Delta}{2 \Omega_{0}}\right)^{2} \sin ^{2}\left(\Omega_{0} t\right)\right]^{2} \\
& +\frac{1}{4}\left[\left(\frac{k}{\Omega_{0}}\right)^{4} \sin ^{4}\left(\Omega_{0} t\right)+2\left(\frac{k}{\Omega_{0}}\right)^{2} \sin ^{2}\left(\Omega_{0} t\right)\right] .
\end{aligned}
$$

In case I $\left(\lambda^{\prime}=\lambda / N\right)$, the coupling strength $k=2 \lambda / \sqrt{N}$ is inversely proportional to the square root of the spin number $N$. In the large $N$ limit,

$$
k^{2}=\frac{4 \lambda^{2}}{N} \ll 4 \lambda^{2}=\Delta^{2},
$$

i.e.,

$$
\begin{aligned}
& \lim _{N \rightarrow \infty}\left(\frac{k}{\Omega_{0}}\right)^{2}=\frac{4 k^{2}}{\Delta^{2}}=0, \\
& \lim _{N \rightarrow \infty}\left(\frac{\Delta}{2 \Omega_{0}}\right)^{2}=\frac{\Delta^{2}}{\Delta^{2}}=1 .
\end{aligned}
$$

Hence from Eq. (28), we have $P=1$ in large $N$ limit. This analytical analysis agrees well with Eq. (22) and Fig. 1.

Generally speaking, the quantum coherence (measured by purity) of a quantum open system would be dissipated by its bath. But when the coupling strength between the system and its bath becomes vanishingly small, and the state of the bath is properly chosen, the system will preserve all its coherence (remains in a pure state or the purity remains to be unity) during the dynamic evolution. In the thermodynamic limit, the coupling strength between the central qubit and the "radiation field" becomes vanishingly small, and the "radiation field" is in low excitation Fock state $|0\rangle$. Thus the central qubit evolves under the free Hamiltonian $H_{S}=-2 s_{z}$, which preserves quantum coherence of the central qubit.

In case II $\left(\lambda^{\prime}=\lambda / \sqrt{N}\right)$, the coupling strength $k=2 \lambda$. Even in the thermodynamic limit, the interaction Hamiltonian does not vanish $\left(2 \lambda^{\prime} \sqrt{N}=2 \lambda \neq 0\right)$. This is why the purity of the central qubit varies in case II even when the bath is in low excitation Fock state $|0\rangle$, which is the ground state of the bath in symmetry broken phase. In this case, the purity (28) can be simplified to

$$
\lim _{N \rightarrow \infty} P=\frac{1}{4}\left[\frac{32}{25} \sin ^{4}(\sqrt{5} \lambda t)-\frac{8}{5} \sin ^{2}(\sqrt{5} \lambda t)+4\right],
$$

which is independent of $N$, as we observed numerically in Fig. 3.

Before concluding this subsection, we would like to emphasize one point. In the symmetric phase $(\lambda<1)$ and in case I $\left(\lambda^{\prime}=\lambda / N\right)$, the above model is mapped into an effective anti-JC model with vanishing coupling strength. However, it should not be regarded that the central qubit and the multispin bath are decoupled. Because there are $N$ bonds between the central qubit and the bath, though the coupling strength $\lambda / N$ goes to zero as $N$ grows large, the overall coupling to the bath will not go to zero.

\section{B. Symmetric phase}

In the above discussion, the system and the bath is reduced into an anti-JC model with a normal coupling, i.e., the coupling does not depend on the mean "photon" number. We now show that the system and the bath can be reduced into the anti-JC model with an intensity-dependent coupling when the bath is in the symmetric phase. In the symmetric phase $(\lambda>1)$, however, the ground state $|N / 2, I(\lambda)\rangle$ of the bath is no longer a low excitation state after the HP transformation. The mean "photon" number

$$
n=\left\langle d^{\dagger} d\right\rangle=\frac{N}{2}-I(\lambda) \approx \frac{N}{2}\left(1-\frac{1}{\lambda}\right)
$$

is of the same order of $N$. By applying HP transformation the Hamiltonian (6) can be rewritten as

$$
\begin{aligned}
H= & \frac{2 \lambda}{N}\left(d^{\dagger} d\right)^{2}+2(1-\lambda) d^{\dagger} d-N-2 \lambda^{\prime}\left(s_{+} d^{\dagger} \sqrt{N-d^{\dagger} d}\right. \\
& \left.+s_{-} \sqrt{N-d^{\dagger} d} d\right)-2 s_{z} .
\end{aligned}
$$

The model described by this Hamiltonian (33) is an intensity-dependent coupling anti-JC model with a Kerreffect term $2 \lambda\left(d^{\dagger} d\right)^{2} / N$, which can be analytically diagonalized as well. After a similar derivation to that in the symmetry broken phase, we obtain the solution $|\Phi(t)\rangle$ of the Schrödinger equation $i \hbar \partial_{t}|\Phi(t)\rangle=H|\Phi(t)\rangle$,

$$
|\Phi(t)\rangle=\sum_{n=0}\left[c_{\uparrow, n+1}^{\prime}(t)|\uparrow\rangle \otimes|n+1\rangle+c_{\downarrow, n}^{\prime}(t)|\downarrow\rangle \otimes|n\rangle\right],
$$

where

$$
\begin{aligned}
c_{\uparrow, n+1}^{\prime}(t)= & \left\{c_{\uparrow, n+1}^{\prime}(0)\left[\cos \left(\Omega_{n}^{\prime} t\right)-i \frac{\Lambda_{n}^{\prime}}{\Omega_{n}^{\prime}} \sin \left(\Omega_{n}^{\prime} t\right)\right]\right. \\
& \left.+i c_{\downarrow, n}^{\prime}(0) \frac{\Gamma_{n}^{\prime}}{\Omega_{n}^{\prime}} \sin \left(\Omega_{n}^{\prime} t\right)\right\} \exp \left\{-i A_{n} t\right\}, \\
c_{\downarrow, n}^{\prime}(t)= & \left\{c_{\downarrow, n}^{\prime}(0)\left[\cos \left(\Omega_{n}^{\prime} t\right)+i \frac{\Lambda_{n}^{\prime}}{\Omega_{n}^{\prime}} \sin \left(\Omega_{n}^{\prime} t\right)\right]\right. \\
& \left.+i c_{\uparrow, n+1}^{\prime}(0) \frac{\Gamma_{n}^{\prime}}{\Omega_{n}^{\prime}} \sin \left(\Omega_{n}^{\prime} t\right)\right\} \exp \left\{-i B_{n} t\right\},
\end{aligned}
$$

and 


$$
\begin{gathered}
\Delta^{\prime}=2(1-\lambda)+(-2)=-2 \lambda, \\
\Lambda_{n}^{\prime}=\frac{\lambda}{N}(2 n+1)+\frac{\Delta^{\prime}}{2}, \\
\Gamma_{n}^{\prime}=2 \lambda^{\prime} \sqrt{(N-n)(n+1),} \\
\Omega_{n}^{\prime}=\sqrt{\left(\Lambda^{\prime}\right)^{2}+\left(\Gamma^{\prime}\right)^{2}}, \\
A_{n}=\frac{\lambda}{N}\left[n^{2}+(n+1)^{2}\right]-\frac{\Delta^{\prime}}{2}, \\
B_{n}=\frac{\lambda}{N}\left[n^{2}+(n+1)^{2}\right]+\frac{\Delta^{\prime}}{2} .
\end{gathered}
$$

The purity of the central qubit [with an initial state $(|\uparrow\rangle$ $+|\downarrow\rangle) / \sqrt{2}]$ in the symmetric phase $(\lambda>1)$ can be determined as (see Appendix B)

$$
P=\frac{1}{2}+\frac{1}{2}\left[1-\left(\frac{2 \lambda^{\prime}}{\Omega_{n}^{\prime}}\right)^{2}(N-n)(n+1) \sin ^{2}\left(\Omega_{n}^{\prime}\right)\right]^{2} .
$$

In case I $\left(\lambda^{\prime}=\lambda / N\right)$, the purity $P(37)$ can be further simplified as

$$
P=\frac{1}{2}+\frac{1}{2}\left[1-\left(1-\frac{1}{\lambda^{2}}\right) \sin ^{2}(\lambda t)\right]^{2} .
$$

We see from Eq. (38) that $P$ varies periodically and is independent of $N$, though the coupling strength $-2 \lambda^{\prime} \sqrt{N-d^{\dagger} d}$ in Eq. (33) depends on $N$, as we have seen in Fig. 1. The physics behind Eq. (38) is that the mean "photon" number of the ground state is also $N$-dependent, which countervails with the $N$-dependent coupling strength, leading to the $N$-independent dynamical behavior of the purity $P$ in case I.

In case II $\left(\lambda^{\prime}=\lambda / \sqrt{N}\right)$, the purity $P(37)$ can be further simplified as

$$
P=\frac{1}{2}+\frac{1}{2}\left\{1-\sin ^{2}\left[\sqrt{N\left(\lambda^{2}-1\right)} t\right]\right\}^{2} .
$$

Hence there does not exist an asymptotic value of the purity when $N$ increases, as observed in Figs. 3 and 4. The $N$ dependence of the purity $P$ in case II stems from the coupling strength $-2 \lambda^{\prime} \sqrt{N-d^{\dagger} d}$ and the $N$-dependent mean "photon" number of the ground state; the $N$ dependence of them cannot countervail with each other.

\section{SUMMARY}

We have studied the dynamic property of a central qubit coupled to an isotropic Lipkin-Meshkov-Glick bath. Two different types of coupling strength between the central qubit and the bath are considered. In both cases, the QPT of the bath is well revealed by the dynamic behavior of the central qubit. We have found that our model is equivalent to an anti-JC model under HP transformation when the bath is in the symmetry broken phase. Especially, when the coupling strength between the central qubit and the bath is inversely proportional to the spin number of the bath, the model can be mapped into an anti-JC model with vanishing coupling strength, and the central qubit preserves its quantum coherence all the time when the bath is in its ground state. The present study not only demonstrates how the QPT influence the quantum coherence of the central qubit, but also establishes the connection between the LMG model and anti-JC model. In addition, our investigation may propose a new scenario to preserve quantum coherence of a central qubit in experimental implementation of quantum computation.

Before concluding this paper, we would like to mention three points. First, our current study is focused on a specific model of the bath and special initial state (the ground state). Nevertheless, our central result (the different behaviors of the qubit when it is coupled to the different phases of the bath) is expected to be generalized to other models of bath with long-ranged couplings, i.e., there is certain universality about our result. Similar universality can be found in shortranged baths (see Refs. [8,24]), where the coupling to the central system impose a perturbation on the QPT controlling parameter of the bath, and the QPT of the bath at quantum critical point is signaled by a sharp decay of quantum coherence of the central system. Second, when the bath is in the symmetric phase $(\lambda>1)$, the choice of initial state (spontaneous symmetry breaking in the bath or not) would have rather large effects on the behavior of the central qubit [6]. We here do not address the spontaneous symmetry breaking of the bath (when it is in the symmetric phase) in our current study. A detailed study on spontaneous symmetry breaking of the model is expected to be done in the future. Moreover, the conjunction between our central result and the main result in Ref. [6] "universal limit to quantum coherence due to spontaneous symmetry breaking" is also interesting and worth to be studied. Third, finite-temperature extension of "QPTinduced decoherence" (Loschmidt echo decay) has been studied in Ref. [25]. Following the same way, it is expected that our result can even be generalized to low but finite temperature.

\section{ACKNOWLEDGMENTS}

This work was supported by the RGC grants of Hong Kong (HKU-3/05C and HKU 7051/06P), and Seed Funding grants of HKU, the National Natural Science Foundation of China under Nos. 10429401, 90203018, 10474104, and 60433050 , and the state key programs of China under Nos. 2001CB309310, 2005CB724508, and 2006CB0L1001.

\section{APPENDIX A: DYNAMICS OF PURITY IN THE SYMMETRY BROKEN PHASE $(0<\boldsymbol{\lambda}<1)$}

For the anti-JC Hamiltonian (24), the time evolution of a initial state $|\Psi(0)\rangle=(|\uparrow\rangle+|\downarrow\rangle) / \sqrt{2} \otimes|0\rangle$ can be expressed as

$$
\begin{aligned}
|\Psi(t)\rangle= & \frac{i k}{\sqrt{2} \Omega_{0}} \sin \left(\Omega_{0} t\right) \exp \left(i \frac{\Delta}{2} t\right)|\uparrow\rangle \otimes|1\rangle+\left[\cos \left(\Omega_{0} t\right)\right. \\
& \left.+\frac{i \Delta}{2 \Omega_{0}} \sin \left(\Omega_{0} t\right)\right] \exp \left(-\frac{i \Delta}{2} t\right)|\downarrow\rangle \otimes|0\rangle+\frac{|\uparrow\rangle}{\sqrt{2}} \otimes|0\rangle .
\end{aligned}
$$

The reduced density matrix $\rho^{S}(t)$ (Eq. (13)) of the system is then found to be 


$$
\begin{aligned}
\rho^{S}(t)= & \operatorname{Tr}_{\mathrm{B}}|\Psi(t)\rangle\langle\Psi(t)| \\
= & \frac{1}{2}\left[1+\left(\frac{k}{\Omega_{0}}\right)^{2} \sin ^{2}\left(\Omega_{0} t\right)\right]|\uparrow\rangle\langle\uparrow|+\frac{1}{2}\left[\cos ^{2}\left(\Omega_{0} t\right)\right. \\
& \left.+\left(\frac{\Delta}{2 \Omega_{0}}\right)^{2} \sin ^{2}\left(\Omega_{0} t\right)\right]|\downarrow\rangle\langle\downarrow|+\frac{1}{2}\left[\cos \left(\Omega_{0} t\right)\right. \\
& \left.-i \frac{\Delta}{2 \Omega_{0}} \sin \left(\Omega_{0} t\right)\right] \exp \left(i \frac{\Delta}{2} t\right)|\uparrow\rangle\langle\downarrow|+\text { H.c. }
\end{aligned}
$$

Applying Eq. (14), we obtain the purity $P$ [Eq. (28)] of the central qubit

$$
\begin{aligned}
P= & \frac{1}{4}\left[1+\cos ^{2}\left(\Omega_{0} t\right)+\left(\frac{\Delta}{2 \Omega_{0}}\right)^{2} \sin ^{2}\left(\Omega_{0} t\right)\right]^{2} \\
& +\frac{1}{4}\left[\left(\frac{k}{\Omega_{0}}\right)^{4} \sin ^{4}\left(\Omega_{0} t\right)+2\left(\frac{k}{\Omega_{0}}\right)^{2} \sin ^{2}\left(\Omega_{0} t\right)\right] .
\end{aligned}
$$

\section{APPENDIX B: DYNAMICS OF PURITY IN THE SYMMETRIC PHASE $(\boldsymbol{\lambda}>\mathbf{1})$}

For a generalized anti-JC Hamiltonian [Eq. (33)], the time evolution of an initial state $|\Phi(0)\rangle=(|\uparrow\rangle+|\downarrow\rangle) / \sqrt{2} \otimes|n\rangle$ can be expressed as

$$
\begin{aligned}
|\Phi(t)\rangle= & \frac{1}{\sqrt{2}}\left\{\cos \left(\Omega_{n-1}^{\prime} t\right)-i \frac{\Xi_{n-1}}{\Omega_{n-1}^{\prime}} \sin \left(\Omega_{n-1}^{\prime} t\right)\right\} \\
& \times \exp \left(-i A_{n-1} t\right)|\uparrow\rangle \otimes|n\rangle+\frac{i}{\sqrt{2}} \frac{\Gamma_{n}^{\prime}}{\Omega_{n-1}^{\prime}} \sin \left(\Omega_{n-1}^{\prime} t\right) \\
& \times \exp \left(-i B_{n-1} t\right)|\downarrow\rangle \otimes|n-1\rangle
\end{aligned}
$$

$$
\begin{aligned}
& +\frac{1}{\sqrt{2}}\left\{\cos \left(\Omega_{n-1}^{\prime} t\right)+i \frac{\Xi_{n}}{\Omega_{n}^{\prime}} \sin \left(\Omega_{n-1}^{\prime} t\right)\right\} \\
& \times \exp \left(-i B_{n} t\right)|\downarrow\rangle \otimes|n\rangle+\frac{i}{\sqrt{2}} \frac{\Gamma_{n}^{\prime}}{\Omega_{n}^{\prime}} \sin \left(\Omega_{n-1}^{\prime} t\right) \\
& \times \exp \left(-i A_{n} t\right)|\uparrow\rangle \otimes|n+1\rangle,
\end{aligned}
$$

where

$$
\Xi_{n}=\frac{\lambda}{N}(2 n+1)+\frac{\Delta^{\prime}}{2} .
$$

In the large $N$ limit, the reduced density matrix $\rho^{S}(t)$ [Eq. (13)] of the central qubit is derived as

$$
\begin{aligned}
\rho^{S}(t)= & \operatorname{Tr}_{\mathrm{B}}|\Phi(t)\rangle\langle\Phi(t)| \\
= & \frac{1}{2}|\uparrow\rangle\left\langle\uparrow\left|+\frac{1}{2}\right| \downarrow\right\rangle\langle\downarrow|+\frac{1}{2} \exp \left[-i\left(A_{n}-B_{n}\right) t\right] \\
& \times\left\{\cos \left(\Omega_{n-1}^{\prime} t\right)-\frac{i}{\Omega_{n}^{\prime}}\left[\frac{\lambda}{N}(2 n+1)+\frac{\Delta^{\prime}}{2}\right] \sin \left(\Omega_{n-1}^{\prime} t\right)\right\}^{2} \\
& \times|\uparrow\rangle\langle\downarrow|+\text { H.c. }
\end{aligned}
$$

Using Eq. (14), we obtain the purity $P$ [Eq. (37)] of the central qubit

$$
P=\frac{1}{2}+\frac{1}{2}\left[1-\left(\frac{2 \lambda^{\prime}}{\Omega_{n}^{\prime}}\right)^{2}(N-n)(n+1) \sin ^{2}\left(\frac{\Omega_{n}^{\prime}}{2} t\right)\right]^{2} .
$$

[1] S. Sachdev, Quantum Phase Transition (Cambridge University Press, Cambridge, UK, 1999).

[2] A. Osterloh, L. Amico, G. Falci, and R. Fazio, Nature (London) 416, 608 (2002); T. J. Osborne and M. A. Nielsen, Phys. Rev. A 66, 032110 (2002); G. Vidal, J. I. Latorre, E. Rico, and A. Kitaev, Phys. Rev. Lett. 90, 227902 (2003); Y. Chen, P. Zanardi, Z. D. Wang, and F. C. Zhang, New J. Phys. 8, 97 (2006); Y. Chen, Z. D. Wang, and F. C. Zhang, Phys. Rev. B 73, 224414 (2006).

[3] H. J. Lipkin, N. Meshkov, and A. J. Glick, Nucl. Phys. 62, 188 (1965); N. Meshkov, A. J. Glick, and H. J. Lipkin, ibid. 62, 199 (1965); A. J. Glick, H. J. Lipkin, and N. Meshkov, ibid. 62, 211 (1965).

[4] R. H. Dicke, Phys. Rev. 93, 99 (1954).

[5] S. L. Zhu, Phys. Rev. Lett. 96, 077206 (2006).

[6] J. van Wezel, J. van den Brink, and J. Zaanen, Phys. Rev. Lett. 94, 230401 (2005); J. van Wezel, J. Zaanen, and J. van den Brink, Phys. Rev. B 74, 094430 (2006); J. van Wezel and J. van den Brink, J. Magn. Magn. Mater. 310, e503 (2007); e-print arXiv:physics/0609177.

[7] S. Paganelli, F. de Pasquale, and S. M. Giampaolo, Phys. Rev. A 66, 052317 (2002); M. Lucamarini, S. Paganelli, and S. Mancini, ibid. 69, 062308 (2004).

[8] H. T. Quan, Z. Song, X. F. Liu, P. Zanardi, and C. P. Sun, Phys.
Rev. Lett. 96, 140604 (2006); D. Rossini et al., J. Phys. A 40, 8033 (2007).

[9] W. H. Zurek, F. M. Cucchietti, and J. P. Paz, e-print arXiv:quant-ph/0312207; Acta Phys. Pol. B 38, 1685 (2007).

[10] M. A. Nielsen and I. L. Chuang, Quantum Computation and Quantum Information (Cambridge University Press, Cambridge, UK, 2000).

[11] D. V. Khveshchenko, Phys. Rev. B 68, 193307 (2003).

[12] M. O. Scully and M. S. Zubairy, Quantum Optics (Cambridge University Press, Cambridge, UK, 1997).

[13] J. Vidal, G. Palacios, and R. Mosseri, Phys. Rev. A 69, 022107 (2004); R. Somma, G. Ortiz, H. Barnum, E. Knill, and L. Viola, ibid. 70, 042311 (2004); S. Dusuel and J. Vidal, Phys. Rev. Lett. 93, 237204 (2004).

[14] S. Dusuel and J. Vidal, Phys. Rev. B 71, 224420 (2005); J. I. Latorre, R. Orus, E. Rico, and J. Vidal, Phys. Rev. A 71, 064101 (2005).

[15] T. Barthel, S. Dusuel, and J. Vidal, Phys. Rev. Lett. 97, 220402 (2006).

[16] F. M. Cucchietti, D. A. R. Dalvit, J. P. Paz, and W. H. Zurek, Phys. Rev. Lett. 91, 210403 (2003); X. S. Ma, A. M. Wang, X. D. Yang, and H. You, J. Phys. A 38, 2761 (2005), and references therein.

[17] X. Z. Yuan and K. D. Zhu, Europhys. Lett. 69, 868 (2005); X. 
Z. Yuan, K. D. Zhu, and Z. J. Wu, Eur. Phys. J. D 33, 129 (2005); X. S. Ma, A. M. Wang, X. D. Yang, and H. You, J. Phys. A 38, 2761 (2005).

[18] A. Olaya-Castro, N. F. Johnson, and L. Quiroga, J. Opt. B: Quantum Semiclassical Opt. 6, S730 (2004); Y. Hamdouni, M. Fannes, and F. Petruccione, Phys. Rev. B 73, 245323 (2006).

[19] L. Tessieri and J. Wilkie, J. Phys. A 36, 12305 (2003); C. M. Dawson, A. P. Hines, R. H. McKenzie, and G. J. Milburn, Phys. Rev. A 71, 052321 (2005).

[20] B. Buck and C. V. Sukumar, Phys. Lett. 81A, 132 (1981); J. Phys. A 17, 885 (1984); V. Buzek, Phys. Rev. A 39, 3196 (1989); X. Wang, Chin. Sci. Bull. 41, 17 (1996).
[21] J. Reslen, L. Quroga, and N. F. Johnson, Europhys. Lett. 69, 8 (2005); G. Liberti and R. L. Zaffino, Eur. Phys. J. B 44, 535 (2005); J. Vidal and S. Dusuel, Europhys. Lett. 74, 817 (2006).

[22] T. Holstein and H. Primakoff, Phys. Rev. 58, 1098 (1940).

[23] G. R. Jin, P. Zhang, Y. X. Liu, and C. P. Sun, Phys. Rev. B 68, 134301 (2003).

[24] F. M. Cucchietti, S. Fernandez-Vidal, and J. P. Paz, Phys. Rev. A 75, 032337 (2007).

[25] P. Zanardi, H. T. Quan, X. G. Wang, and C. P. Sun, Phys. Rev. A 75, 032109 (2007). 